Fecha de recepción: octubre 2021

Fecha de aprobación: noviembre 2021

Fecha publicación: diciembre 2021

\section{Cartografía de una hibridación para desplazados: la obra de Lucy + Jorge Orta (1992-2012)}

Dra. Ana Llorente Villasevil ${ }^{(1)}$

Resumen: Este artículo tiene como objetivo evaluar el acercamiento al diseño indumentario por parte del tándem artístico formado por Lucy y Jorge Orta para el desarrollo de prototipos de refugios portables que nacieron hace treinta años con el fin de cubrir necesidades de refugio en situaciones de indigencia y migración. Se revisará la base operativa y funcional de una obra que se desarrolló en paralelo a un ambiente de reflexión y actuación que, gracias a creadores como Tess Giberson, Vexed Generation o Hussein Chalayan, transitaba por la esfera del arte, la moda y la arquitectura por medio de diferentes parámetros de actuación.

Palabras clave: diseño de moda - hibridación - diseño arquitectónico - transdisciplinar refugio - indigencia - migración - prototipos - portable.

[Resúmenes en inglés y portugués en las páginas 168-169]

(1) Licenciada en Historia y Teoría del Arte. Universidad Autónoma de Madrid. Máster en Gestión del Patrimonio Cultural, Turístico y Natural. Fundación Ortega y Gasset. Madrid. Doctora-Cum Laude. Universidad Autónoma de Madrid. Profesora Escuela Universitaria de Diseño, Innovación y Tecnología, Madrid y otras instituciones.

\title{
Introducción
}

La diversidad de grupos nómadas y seminómadas, los territorios en los que se mueven y los motivos de su condición provocan diferentes modos de negociar la satisfacción de la necesidad de refugio. Durante milenios el dominio a este nivel lo han tenido las soluciones puramente arquitectónicas con construcciones temporales, móviles y adaptables. No obstante, en las tres últimas décadas no solo se ha renovado un interés por la evolución de tales tipologías arquitectónicas, sino que estas se están abriendo a una inseminación por parte de otros lenguajes del diseño. De entre ellos, el indumentario ha conformado el terreno más fértil para crear hibridaciones con las que aportar soluciones que nutran un acuerdo entre la protección física y las relaciones con el entorno de individuos y comunidades desplazadas. 
Desde la década de los noventa, estos campos de experimentación se consolidaron a consecuencia de la paulatina intensidad de crisis migratorias como resultado de desastres naturales y conflictos bélicos. Dentro de este marco emerge un estadio inicial de la obra del tándem formado por la artista plástica y visual británica Lucy Orta y el arquitecto argentino Jorge Orta, fundadores de Studio Orta. Sus prototipos híbridos dotados de la capacidad de operar alternativamente como prenda y como construcción nacían en paralelo a un ambiente de examen y reflexión que, en años sucesivos, transitaba por la esfera del arte, la moda y la arquitectura por medio de diferentes parámetros de actuación. Así, por ejemplo, el proyecto de investigación dirigido en la Universidad de Liverpool por Robert Kronenburg, y conocido como Portable Architecture, daría lugar a una homónima exposición celebrada en 1998 por el Royal Institute of British Architects de Londres donde se recogerían, entre otros trabajos, modelos de refugios temporales para circunstancias adversas. En ese mismo año, Aedes East Gallery de Berlín reunía la obra de la diseñadora Yeohlee Teng y la del arquitecto Ken Yeang, cofundador de la firma T. R. Hamzah \& Yeang en Energetik: Kleidung und Umkleidungen. Esta exposición descubriría cómo los dos creadores malasios usaban combinaciones de cerramientos textiles, indumentarios y arquitectónicos, para perseguir dentro sus áreas respectivas la aclimatación efectiva del ser humano que viste y habita en constante traslación por diversos entornos. Dos años después, Hussein Chalayan se abriría a la hibridación con otros lenguajes del diseño, para embeber su creación de narraciones comprometidas con la realidad del desplazamiento y exilio por conflictos bélicos, así como con estados de vida aislada e itinerante vinculados a la era del progreso tecnológico.

Frente a todo ello, el trabajo de los Orta parecía evidenciar una adelantada posición por parte de la esfera artística en el aprovechamiento de la cualidad indumentaria para el desarrollo de prototipos de refugios portables que funcionan como ropa, y viceversa, destinados a situaciones de indigencia y migración. Estas creaciones han sido objeto recurrente de exposición y de análisis como dispositivos de supervivencia ya no tanto por medio de la provisión de protección física, sino de emplazamiento y conexión social (Orta, 2003a; Orta, 2003b; Pinto, 2003; Quinn, 2003; Restany, 2001). Igualmente, se han abordado en sintonía con los diseños de Tess Giberson, Vexed Generation o Hussein Chalayan; esto es, como partes de un paisaje de prácticas performativas destinadas igualmente a la reinscripción del sujeto en el dominio urbano, que se antoja catalizadora de la integración y pertenencia a un lugar (Johung, 2013; Martín Asunción, 2012).

En el presente escrito se revisará la base operativa y funcional de la obra de Lucy y Jorge Orta contando con nuevos referentes para guiar su interpretación. Se evaluará a partir de la perspectiva ofrecida por modelos de suministro de refugio y métodos de organización comunitaria llevados a cabo por organizaciones como Oxfam o The Mad Housers.

\section{Consideraciones en torno a la definición filogenética del arropamiento y la construcción: de la agencia manual a la corporal}

A pesar de que la moda y la arquitectura suelen entablar diálogos creativos que no superan niveles de poética visual articulada en la mayoría de las ocasiones por medio de semejan- 
zas formales entre la prenda y el edificio, se distinguen dos puentes básicos de conexión. En primer lugar se encuentran enlaces soportantes: conectores materiales textiles fundamentalmente, procesuales y tecnológicos que vehiculizan la toma de contacto de los dos sectores creativos en campos de diálogo y patrones de interacción. En segundo lugar se sitúan los enlaces creativos que pueden resumirse como puentes conceptuales y funcionales que residen en el origen de la creación de un diseño, y que son responsables de añadirle valor simbólico y sociocultural. Así, por ejemplo, a través del principio de adecuación o conveniencia del edificio al propietario, el arquitecto opera en el mismo régimen de jerarquización visual del que comúnmente es partícipe la ropa.

De entre los enlaces funcionales, la necesidad de cobijo es la raíz o núcleo genérico potencialmente capaz de dar instrucciones para el desarrollo efectivo de una fecundación entre la ropa y la edificación. No es de extrañar que las hipótesis sobre los orígenes de la construcción y la cubrición corporal han interceptado en el pensamiento de ambas como resultado de la necesidad instintiva de amparo físico en un entorno no dominable.

Eco notó que la sucesión de estímulos y eventos sensoriales que habrían conducido al ser humano a aprehender como cobijos las cuevas naturales para conformar el código arquitectónico es aplicable a la consecución de la "proyección vestimentaria" (2011, p. 253).

A este último respecto basta observar cómo en situaciones de emergencia, los materiales disponibles pueden ser tan limitados que el ser humano multiplica la aplicabilidad de los recursos con operaciones que pueden hacer pasar una cubrición corporal a la arquitectónica, y viceversa. Como recordaba Bernard Kerblat (1985), jefe de la sección de preparación y respuesta en emergencias del Alto Comisionado de las Naciones Unidas para los Refugiados, así hicieron con simples trozos de plástico comprados en mercados locales los refugiados masivos víctimas de los conflictos bélicos que sostuvo Vietnam con Camboya y China a finales de los setenta. Observar cómo este recurso les permitía desde arropar el cuerpo a la intemperie a construir un refugio con dos palos de bambú, habría impulsado la homologación en 1985 de las plastic sheeting por Naciones Unidas. Usadas en la supervivencia, así como en la dignificación de la vida y la muerte, las láminas son un ejemplo del vínculo filogenético entre la cubrición corporal y el refugio. La presión del contexto y la respuesta adaptativa conducirán a las transformaciones de una a otra, que se revelan con claridad como la operación de lo bidimensional a lo tridimensional, y viceversa.

Desde la década de los noventa, estos estadios de transición han sido examinados como agentes de fertilizaciones del lenguaje indumentario y el arquitectónico por Sophia Vyzovity. Como parte de sus proyectos de investigación en la Universidad de Tesalónica, la arquitecta comenzó a llevar a cabo experimentos a pequeña escala manipulando materiales flexibles y potencialmente polimórficos como el papel y la gomaespuma. Con gestos espontáneos eran deformados para generar lo que llamó paperfolds; estos son formas primarias que pueden ser desarrolladas hasta configurar prototipos para diferentes tipos de productos, desde edificios a sillas (Vyzoviti, 2003). En la traslación de esta intuitiva experimentación manual con el material a escala humana, Vyzovity hizo pasar al cuerpo desde una actuación externa a las formas hasta ejercicios de modelado desde el interior. El sujeto pasa a establecer una relación de presencia y de ocupación con el material. Así ha sucedido para la generación de los prototipos multigarments, body wraps o body donuts. 
Este proceso de generación formal mantiene un indeterminismo creativo que permite deslizar la identidad del objeto a concebir hacia un estado entremedias de la construcción y la ropa, difuminando las fronteras entre la habitación y el hábito. La implicación física con materiales flexibles demuestra el desencadenamiento de una pulsión instintiva de cubrición corporal, trasladando a niveles de ideación previos a la conclusión del diseño lo que la realidad demuestra con la experiencia de las láminas de plástico para desplazados. En cierta manera, ya lo había advertido Feuerstein al afirmar que "la unión de arquitectura y ropa sucede en el momento en que se comprende una necesidad común en el cuerpo y el edificio; esto es, la cubrición de su forma desnuda" (1988, p. 17).

Ahora bien, si en estadios experimentales de generación formal, así como en aplicaciones de una simple hoja de plástico en estados de desamparo y migración, se opera naturalmente en la transferencia de propiedades del ámbito indumentario al constructivo, ¿hasta qué punto puede concluirse una fecundación efectiva entre el diseño arquitectónico y de ropa?, cabe plantearse si la prenda puede complementar sus aptitudes como protector corporal, incorporando cualidades estructurales que le permitan dar asentamiento en un lugar por un tiempo limitado sin perder su cualidad móvil. Los resultados de la suma deberían tener un potencial práctico, fruto de un equilibrio entre los dos lenguajes y adecuado a los fines múltiples -básicamente, protección, localización, acotación espacial y ambiental, visibilidad y movilidad.

\section{Cuatro coordenadas para una localización de los refugios portables: desplazamiento, conexión, intervención e interrupción}

En 1992, Lucy y Jorge Orta presentaron la serie titulada Refuge Wear, tomando como detonante las necesidades de los refugiados kurdos iraquíes, desarraigados y desplazados a causa de la Guerra del Golfo (1990-1991). Los primeros modelos consistían en anoraks que cubrían al sujeto de la cabeza a los pies y que podían convertirse reversiblemente en tiendas de campaña. Constaban de una estructura ligera de carbono sobre la cual se tensaba la tela de la prenda. Dicho tejido era de fibras naturales tanto como tecnológicas de alto rendimiento. Algunos de los prototipos de esta serie quedaban además revestidos a través de serigrafías de mensajes textuales y visuales que pretendían llamar la atención sobre la condición del individuo desplazado.

A las unidades de refugio individual que compusieron Refuge Wear, proyecto en el que estuvieron trabajando hasta 2007, se solapó el desarrollo de otros trabajos como Nexus Architecture (1993-2002), consistentes en monos encapuchados que permitían conectar a la altura de la espalda y el estómago a los sujetos que los visten mediante tubos de tela unidos al traje por una cremallera. Dentro de esta idea de modularidad interconectada se desarrolló también Collective Wear Body Architecture (1994-1999). Los diseños de esta última serie se destinaban a dar habitación y ropa a los sin techo gracias a módulos convertibles reversiblemente en tienda de campaña y prenda. Pero además añadían la opción de conectar entre sí cada unidad individual para formar núcleos de población entre personas que desearan vivir en comunidad. Este es el caso de las series Mobile Villages con obras 
como Connector Mobile Village (2002), expandible con tantas posibilidades residenciales, sociales o funcionales como módulos fuesen conectados.

Los prototipos de Lucy y Jorge Orta se podrían relacionar con ciertos trabajos del artista alemán Franz Erhard Walther. Concretamente, en Werksatz (1963-1969 y 1972-1973) los espectadores son invitados a implicarse en un proceso cognitivo y de interacción con el grupo dirigido a través de los Benutzbare Objekte objetos para ser usados; esculturas textiles que eran medios que dirigían al sujeto desde, la experiencia individual de vestir el cuerpo como vía de ocupación y comunicación con el espacio hasta la conectiva con la comunidad, generando al final las condiciones de un espacio pre-arquitectónico.

En cualquier caso, el principal punto de unión de la obra de los Orta y la de Franz Erhard Walther, este sería el de la intervención en el dominio público guiada por el activismo ideológico. El carácter seminal del trabajo de Lucy y Jorge Orta residió en sus Interventions, basados en la ocupación de los espacios urbanos para testar al tiempo que exhibir los diseños pertenecientes a series como Nexus o Refuge Wear con voluntarios del Ejército de Salvación de Londres, tanto como con personas sin hogar en ciudades como Nueva York, La Habana o París. Como declaró la artista británica al crítico y comisario Nicolás Bourriaud (2003), en un primer momento, el destino de su trabajo no residía en el espacio de museos o galerías, sino directamente en el paisaje urbano como catalizador de la unión de su investigación con la práctica.

Estas inquietudes entraron en sintonía con las que estaban movilizando a grupos coetáneos de acción social como The Mad Housers. Estos jóvenes de Atlanta, algunos de ellos graduados en arquitectura, se habían convertido a finales de los ochenta en un fenómeno al construir ilegalmente alrededor de cincuenta y cinco pequeñas cabañas para individuos y personas sin hogar. Los Orta coincidieron con este colectivo en intentar llevar a cabo una puesta en práctica de sus creaciones dentro del espacio urbano. Lo que marca la diferencia frente a lo que fueron consideradas acciones de desobediencia civil por parte de los de Atlanta es que la obra del tándem elude el trabajo sobre construcciones permanentes. El establecimiento no es una opción para la función de los diseños híbridos de Lucy y Jorge Orta. El sujeto y el grupo a los que se destinan sólo permanecen en el tránsito. Antes que a la propia condición de indigencia o desplazamiento, este problema se debe a restricciones legales de larga trayectoria.

Los asentamientos con tiendas de campaña o tent cities como solución para la gente sin hogar datan de 1949 cuando, tras la Segunda Guerra Mundial, Henri-Antoine Groue, conocido como el Abad Pierre, consiguió reunir por llamamiento popular trescientas tiendas que acabaron ocupando las orillas del río Sena en París. Dos años después fueron retiradas por el gobierno. Desde entonces, a lo largo de ciudades por todo el mundo, incluyendo París con el campamento de los SDF o Sans Domicile Fixe, asentado y desmantelado en el canal Saint-Martin en 2006 se ha tratado de implementar la utilización de la tienda de campaña como medio racional para dar alojamiento fijo y seguridad a las personas sin hogar.

Dada la imposibilidad de asentamiento estable, la traslación se hace imprescindible; todo lo cual justifica la implicación del diseño y confección de ropa, lenguaje en el que Lucy Orta se había formado, como vía de transformación de la habitación en hábito. Sus intervenciones se convierten así en interrupciones de las narrativas acostumbradas en los espacios públicos para generar hiatos en la experiencia urbana diaria con situaciones ex- 
céntricas que cuentan con el nivel vestimentario como medio de portabilidad y de visibilidad de lo periférico.

Cinco años después de la producción de los primeros modelos de Refuge Wear, la diseñadora Yeohlee Teng trasladaba la moda a este último terreno conceptual. Su colección de otoño/invierno de 1997 se dirigía a los urban nomads, grupo sometido a migraciones debidas a un marco socioeconómico que le obliga a transitar diariamente por diferentes entornos climáticos y atmosféricos, como los interiores de edificios que conforman una suerte de quinta estación (Martin, 1998). Con todo, Yeohlee situó el paradigma de la prenda como arquitectura íntima en unas coordenadas distintas a las de los Orta. En su función estabilizadora de las sensaciones del cuerpo que se mueve por ambientes cambiantes, su obra se sostuvo en un mero sistema de cubriciones que podía poner o quitar según correspondiese. Esto permitió a la exposición de 1998, Energetics: Clothes \& Enclosures, establecer una ligazón con los mecanismos que rigen el diseño bioclimático de arquitectos como Ken Yeang, creador de fachadas como la de Menara Mesiniaga Tower con dispositivos sensibles a la luz, la temperatura y la humedad exterior.

Para la diseñadora malaya, el nomadismo es un modo de vida manifiesto en todo patrón de traslación. "Anybody who goes from point A to point $B(\ldots)$ in the course of a day or a lifetime, is a nomadic creature" (Koda, 2003, p. 93). Tal generalización no adscribe necesariamente su filosofía creativa a marcos críticos y a la lógica de supervivencia que, tradicionalmente, ha justificado el no asentamiento de grupos humanos. Ahora bien, envuelto en el desarrollo de una vida laboral, se podría entender que el sujeto de Yeohlee es víctima de un nomadismo edulcorado por un sistema que normaliza la movilidad frente al establecimiento como medio de sometimiento a un estado constante de precarización.

\section{Cuatro dominios para una deslocalización de los refugios portables: indeterminación, transformación, agencia y reflexión}

Sea como fuere, el usuario de la ropa de Yeohlee no es nómada, sino seminómada. Al finalizar el día, tiene refugio, bien en el estable del hogar, bien en el temporal de un hotel. Esto hace que sus prendas no precisen de un potencial edificatorio. No pierden su naturaleza como ropa, pudiendo ser inscritas en el contexto comercial de la moda, contrariamente a lo sucedido con las creaciones de los Orta, que adolecen de un constante estado de indeterminación formal en uno $\mathrm{u}$ otro nivel de existencia indumentario y arquitectónico. Siempre empleando tipologías encapuchadas, si se contemplan modelos como Model Survival (Refuge Wear, 1996-2006), apenas se reconocen como prenda, ni siquiera vestidas. Su identificación lleva asociada una cierta tensión, hasta el punto de ser percibidos como cualquier otro artilugio -frecuentemente, sacos de dormir. Incluso en su estadio como atuendo, un modelo como el Mobile Cocoon with detachable Baby Carrier (Refuge Wear, 1994) suscita una extrañeza semejante a la que provocan los monos de protección quími$\mathrm{ca}$, en los que poco o nada se ve del sujeto que los viste. Funcionalmente, tienden a esconder la identidad y castran así la necesidad psicogenética de reconocimiento y aceptación social. Pero en la ocultación se hace visible lo invisible. 
Es en este punto donde el trabajo de Lucy y Jorge Orta sintoniza con la vía apuntada por Christo y Jeanne-Claude quienes impulsaron la apreciación de una relación del edificio con la ropa a través de su envolvente textil que tan temporal, pero suntuosamente, cubría la arquitectura soporte de sus intervenciones artísticas. De hecho, es interesante la proximidad cronológica de los diseños de los Orta con uno de los trabajos más famosos del matrimonio, el Wrapped Reichstag (1995), que consistió en mantener envuelta durante dos semanas con tela plateada y cuerdas azules la superficie del imponente edificio neorrenacentista del siglo XIX, sede del Bundestag. El tejido con el que se empaquetan o envuelven este y otros edificios es el que acaba revelando la esencia de los mismos. Del mismo modo, envolventes corporales como las de los Orta, cuyas propiedades visuales apelan a un sentido de extrañeza al alejarse de las comunes en la indumentaria, son instrumentos de subjetivación del individuo que las viste, aunque solo sea por llamar la atención sobre su presencia en el paisaje urbano.

Aunque más definido, su estadio arquitectónico genera una confusión similar quedando visibles los remanentes vestimentarios tales como brazos y piernas colgando a lo largo de la superficie de las construcciones temporales. Por ejemplo, Habitent (Refuge Wear, 19921993), un híbrido entre prenda y refugio temporal compuesto por poliamida recubierta de aluminio, lana, y postes telescópicos de aluminio para su montaje, se presenta como una tienda de campaña coronada por una capucha. Con todo ello, es posible localizar estos prototipos en un limbo similar al que Quinn adjudicó a "toda prenda transformable oscilante entre un estado de deconstrucción el desprendimiento de las piezas para descubrir la prenda y de reconstrucción proceso que resaltaría la edificación poniendo el acento en completarla" (2002, p. 120). Por encima de cualquier tensión morfológica e incluso funcional, lo importante es que los diseños híbridos encuentran un punto crítico de definición en la transformación reversible de la indumentaria como construcción. En este acto se concentra la negociación con el entorno acotando un espacio individual o compartido, según corresponde al prototipo. Montando y desmontando, construyendo y confeccionando, habitando y vistiendo, el control que estos artefactos otorgan al sujeto sobre su ocupación y movilidad en el espacio es equiparable a la labor que The Mad Housers hacía al adoctrinar a los sin techo de Atlanta sobre cómo edificar una casa.

Otorgar control a la persona o población en la construcción de su refugio es algo con lo que también trabajan organizaciones humanitarias. Ya en la década de los setenta, Jim Howard y Ron Spice (1989) habían publicado para Oxfam una guía para el uso de las láminas de plástico donde se catalogaron varias clases de tiendas de campaña que podían ser compuestas por quienes las necesitasen gracias al suministro en kits de elementos de soporte y sujeción como palos y cuerdas. Veinte años después, la Office for the Coordination of Humanitarian Affairs publicaba la propia, donde dejó claro que, "A pesar de que el uso de tiendas para los asentamientos temporales fuese una decisión tomada por las autoridades y organizaciones de apoyo, en la medida de lo posible era indispensable la participación de la gente" (2004, p. 8).

Cabe señalar que Lucy y Jorge Orta ponen el acento sobre el control en la fabricación a través de la organización de proyectos como Identity + Refuge (1995), que partió de la organización de talleres donde personas sin hogar residentes de la Cité de Refuge de París aprendieron a confeccionar un guardarropa a partir de prendas sobrantes en los almace- 
nes del refugio. Estas acciones no solo impulsan la ruptura con la frecuente condena a la inacción en la situación de espera que condicionan ciertos estados como el de los refugiados. También propicia la socialización; objetivo que persigue el proceso transformacional de obras como Body Architecture. Después de quitarse sus trajes, estas personas, reunidas por casualidad o en un área designada, los cierran juntos para hacer una carpa espaciosa para cuatro personas, para, al día siguiente o durante la noche, desabrocharla y meterse en sus trajes para continuar su viaje (Restany, 2001).

Ahora bien, a diferencia de lo que puede suceder con los ejercicios unidireccionales de emplazamiento, establecimiento y socialización de corporaciones como The Mad Housers, los impulsados por el uso de estos prototipos que integran un estadio de ropaje hacen de la transformación del artefacto un acto que alumbra la desintegración social (Orta, 2003). Ya cualquier participación activa del sujeto en la fabricación de su habitación sea permanente, en el caso de las prácticas de los Mad Housers, o temporal, en el caso de las de los Orta o de organizaciones como Oxfam evidencia y detona la reflexión acerca de la falta de patria o de hogar. Pero esta situación se acentúa en el híbrido cuya conversión, como si se tratase de un ritual, refleja la carestía primaria a la que intenta hacer frente su naturaleza adaptativa. Mientras que en la transmutación del sujeto vestido en sujeto alojado se hace inteligible la necesidad de alojamiento, el paso inverso descubre su condición sin techo e incide de nuevo en la carencia de un hábitat estable y permanente.

De esto último se deduce que la matriz de estas hibridaciones es la arquitectura, que no se deja de apreciar como lengua madre en las fases transformativas por las que pasan. Aunque otorgan visibilidad al refugiado, migrante o indigente, como ocultadoras prendas encapuchadas ejercen una función sustitutiva del cobijo arquitectónico sobre el cuerpo en tránsito. La ropa se presenta así como un doble de la construcción, perdiendo identidad como indumentaria. No es de extrañar que en el momento en que se emprendió la producción industrial de Habitent (1992-1993), la versión resultante pareció víctima de una depresión endogámica por la incapacidad del prototipo de operar adecuadamente en su estado como prenda. Este hecho disuadió a Lucy Orta de otros intentos de fabricación masiva de estos modelos (Johung, 2013) y explica la decisión de recluir su trabajo en el discurso artístico.

\section{La reinscripción como conclusión}

La experiencia frustrada de producción masiva de Habitent enseña, en primera instancia, que la prioridad en las circunstancias planteadas para su uso era la de proveer de un refugio temporal de emergencia. La adición de movilidad a la estructura mediante su conversión en prenda no es solo un aspecto secundario, sino ineficaz en términos de producción y uso. Por lo demás, cuestiona de lleno la viabilidad del prototipo híbrido de la edificación y la vestimenta, e invita a rastrear la trayectoria de estas prácticas.

Retóricas explícitas acerca de la función social de una práctica conjunta del diseño y construcción de refugios portables y la creación y confección de ropa llevan a preguntarse si la moda y la arquitectura están avanzando hacia cualquier objetivo por la senda de 
la fertilización mutua. Así pareció interpretarlo en 2006 la exposición comisariada por Brooke Hodge, Skin + Bones: Parallel Practices in Fashion and Architecture, inaugurada en el Museum of Contemporary Art de Los Ángeles (2006). Esta muestra contó con 200 obras de 46 arquitectos, artistas y diseñadores de moda clasificados según un criterio taxonómico fundado en puntos de confluencia como Shelter donde se incluyó la obra de Lucy y Jorge Orta, Tectonic Strategies o categorías culturales como Construction, Reconstruction, Deconstruction.

En concreto, el espacio Synthesis delató cómo la suma de los dos lenguajes del diseño era una idea peregrina para el proyecto curatorial. Modelos expuestos como el Custom Dress (2006), un vestido corto en algodón y poliéster con cola trasera diseñado por Elena Manferdini, tan solo confirman la existencia de medios compartidos por la moda y la arquitectura, tales como el sistema de corte láser o el software Autodesk Maya, lo mismo que sucedería con otras creaciones como Bone Dress de 2008. Así, la noción de síntesis infecta con la contradicción una narrativa expositiva que acabó advirtiendo únicamente que la moda y la arquitectura son sectores que llegan a maniobrar con conceptos o materiales similares, sin romper por ello sus propios marcos de creación, producción, distribución, uso y consumo.

Por su parte, en 2010, Amsterdam Centre for Architecture reunió a cuatro tándems, compuestos por un diseñador de ropa y un estudio de arquitectura. El objetivo era hacer trabajar a cada uno en un proyecto bajo la creencia de que tan solo desde la confrontación directa se podrían desvelar los puntos en los que ambos lenguajes interseptan (Kloos, 2010). Los resultados fueron cuatro vestidos exhibidos en la exposición Fashion \& Architecture que traducen edificios creados por los correspondientes estudios de arquitectura. Así sucedió con el trabajo desarrollado por Benthem Crouwel Architekten y la diseñadora de moda Iris Van Herpen. Se trató de un modelo en plexiglás inspirado en la fachada del New Stedelijk Museum (2012). El proyecto de ARCAM representó la promoción desde una institución cultural de un modelo de colaboración interdisciplinar que constataba el reconocimiento apriorístico de la compatibilidad de la moda y la arquitectura en prácticas no ya paralelas sino conjuntas. Prometía cuanto menos un ejemplo de la constitución de una cultura cruzada del diseño que se dirija al enriquecimiento paralelo de las dos esferas. Pero la moda acabó situándose como campo dominante, escapando de sus modos de presentación para apropiarse mediante esta práctica participativa de los de la exhibición artística. Qué duda cabe que el museo y la galería permiten reinscribir y comprometer las fertilizaciones en un sistema que legitima la inviabilidad práctica de sus resultados mediante su inserción en el dominio de lo simbólico. Basta comprobar cómo decenas de exposiciones han redimido las colaboraciones de Hussein Chalayan con diseñadores industriales como Paul Torpen o estudios de ingeniería como los londinenses $B$ Consultants, de las que resultaron modelos puramente icónicos como Aeroplane Dress, perteneciente a la colección Before Minus primavera/verano del 2000. Este vestido por control remoto, realizado a partir de un molde en fibra de vidrio y resina de color rosa, encerraba el potencial de cambiar su forma a algo parecido a un aeroplano. Pero aquello que debería ser móvil, retiene. De hecho, en el desfile en el Sadler's Wells Theater de Londres donde se presentó Before Minus, el contrapunto al sistema del Aeroplane Dress acaba siendo la sujeción de su aparente funcionamiento a un niño que accionaba por control remoto el despliegue de las solapas 
laterales y traseras de la falda consumando una mera simulación de un vehículo que no responde a las necesidades de motricidad del individuo. Esta idea entra en sintonía con la inmovilidad provocada por el peso simbólico de la pertenencia emocional a un lugar representado por artefactos como la mesa-falda de la famosa Afterwords otoño/invierno de 2000/2001, inspirada en los migrantes chipriotas que en 1974 huyeron con la invasión turca de la isla de Chipre.

Al sumar rasgos de otros objetos a la identidad de su ropa, sin duda, Chalayán la dotó de una naturaleza sintética en términos formales y semánticos. Pero en el camino al estadío hibridacional, su obra va sumando barreras que impiden la consumación del carácter práctico de los prototipos a los que Chalayan acaba denominando "monumentos a las ideas" (Bolton, 2016, p. 111). Su experiencia como mercancías se neutraliza, lo cual facilita su función como transmisores de conceptos que redefinen la relación de la moda con la realidad.

El frecuente confinamiento al museo y la galería de prácticas de apropiación, colaboración y fertilización del diseño arquitectónico e indumentario pone bajo sospecha la posibilidad de superar un nivel de hibridación semántica para alcanzar la pragmática. Incluso con sus intervenciones en diversas ciudades del mundo, los trabajos de Lucy y Jorge Orta quedaron recluidos en un terreno de acción performativa artística. Cierto es que sus cobijos con función dual, a priori, de emplazamiento y desplazamiento, de localización y portabilidad, han sido agentes útiles para la reflexión, aún más cuando eran activados para demandar el derecho de ocupación del dominio urbano como espacio legítimo de vivienda y representación ciudadana. Sin embargo, como la población a la que va dirigida, la obra de Lucy y Jorge Orta, conocedora de sus orígenes, ha transitado por entornos diferentes que plantean la duda sobre su destino.

Durante dos décadas, el control sobre los encargos, la financiación y el desarrollo de estos trabajos lo han tenido instituciones como la Bienal de Johannesburgo, el Festival Histórico Anual de México, para el que crearon Connector Guardian Angel (2001) con la colaboración de pintores del distrito Zócalo de México D.F., o galerías de arte como Lothringer 13 de Múnich. Durante este periodo concreto la moda se ha asegurado un puesto como un activo más del tablero del arte con ejercicios de patronazgo y comisariado. Así, fue precisamente el mecenazgo de Ermenegildo Zegna a través de ZegnArt el que posibilitó el desarrollo del último proyecto en el que se hace partícipe el corpus que se ha tratado en el presente texto. Se trata de Fabulae Romanae en 2012, una instalación producida por Lucy y Jorge Orta empleando varios lenguajes: performativo, fílmico, escultórico, textil, indumentario y arquitectónico. Expuesto en MAXXI Museo Nacional de las Artes del siglo XXI, lo que se antoja un intento de explorar la identidad creativa como un todo a través de distintos medios discurre en un universo desplegado en las entrañas de Roma a través de silenciosos personajes, como el Viajero, el Observador o el Hombre Volador, que transitan como genios locales caracterizados parcialmente en un mediometraje por su vestuario y por la poesía de Mario Petrucci (Studio Orta, 2013). A pesar de la intención de examinar ideas como el peso de la marginalidad, en este marco narrativo fabular los refugio-prendas de los Orta figuran como comparsas, habiendo mutado en tiendas con prendas colgantes a modo de una versión de las arquitecturas modulares que habían formado los Body Architecture. Es inevitable apuntar que en la pérdida de fuerza de los híbridos de Orta, al 
menos como signos de advertencia sobre realidades sociales concretas, media la sintaxis con la que la moda legítima comúnmente su capacidad para producir un capital creativo equiparable al de otros lenguajes.

Frente a ello, queda claro que los sectores académicos conforman las plataformas adecuadas de experimentación y evolución a este nivel. Consolidando lo textil como la interfaz del sujeto con los entornos, este marco empírico sirve por lo pronto para el aislamiento del gen común en el cobijo visualizándolo, como se ha explicado con Sophia Vyzoviti, en la integración activa de lo corpóreo en ejercicios de generación formal. A su vez, la promoción de una formación transdisciplinar apoya la integración en estadios seminales de la creación arquitectónica de conceptos, materiales y técnicas de confección provenientes de la moda y el diseño de ropa, con la finalidad de investigar la creación de modelos que, sin salir del marco de lo estrictamente edificatorio, contengan propiedades y funciones inherentes a lo indumentario. Pero la complejidad del contexto contemporáneo requiere que posiciones oblicuas se consoliden en una alianza más segura y estable de la creación arquitectónica e indumentaria. La fatal actualidad que ha llegado a los más de 15 millones de refugiados y desplazados desde 2015, año de celebración de la Conferencia sobre la Migración y Ciudades (CMC), sumados a los 880 millones de personas que no viven en asentamientos estables en el mundo según datos ya de 2017 (UN-Hábitat, 2017), convierte a la tipología del refugio portable en un asunto central que puede ser aprovechado como oportunidad para sacar adelante prototipos eficientes en la producción, distribución y uso.

\section{Lista de Referencias Bibliográficas}

Bourriaud, N. (2003). Nicolas Bourriaud in correspondence with Lucy Orta. En R. Pinto, N. Bourriaud, L. Orta, \& M. Damianovic. Lucy Orta. Nueva York: Phaidon.

Eco, U. (2011). La estructura ausente. Introducción a la semiótica (Francisco Serra Cantarell, trad.). Buenos Aires: Debolsillo.

Feuerstein, G. (1988). Visionäre Architektur. Frankfurt: Wissenschaften

Howard, J.y Spice, J. (1989). Plastic sheeting: its use for emergency housing and other purposes. Oxford: Oxfam.

Johung, J. (2013). Replaceable Skins: Clothing and Mobile Home. En J. Johung \& A. Sen (eds.). Landscapes of Mobility: Culture, Politics, and Placemaking. Wisconsin: Routledge.

Kerblat, B. (c. 1985). UNHCR United Nations High Commissioner for Refugees. UNHCR Plastic Sheeting (Entrevistador anónimo) [Archivo de Audio]. Recuperado de https:// www.moma.org/multimedia/audio/20/501

Kloos, M. (2010). Fashion \& Architecture, guía de exposición. Amsterdam: ARCAM.

Koda, H. (2003). Fashioning Thoughts. En J. S. Major y Y. Teng (Eds.). Yeohlee: Work. Material architecture. Melbourne: The Image Publishing Group.

Martin, R. (1998). Energetics: Clothes \& Enclosures. Berlin: Aedes Galerie für Architektur und Raum.

Martín, I. (2012). Trajes espaciales. La vestimenta como proyecto arquitectónico [Tesis doctoral, Universidad Politécnica de Madrid]. Repositorio UPM. oai:oa.upm.es:14913 
Office for the Coordination of Humanitarian Affairs [OCHA] (2004). Tents. A guide to the use and logistics of family tents in humanitarian relief. United Nations Publication. Recuperado 11 de noviembre 2019, de https://www.sheltercluster.org/sites/default/files/ docs/Guide\%20to\%20the\%20Use\%20and\%20Logistics\%20of\%20Family\%20Tents.pdf Orta, L. y Restany, P. (1998). Lucy Orta: Process of transformation. Paris: Jean-Michel Place. Orta, L. (2003a). Interview with Andrew Bolton (extract). En R. Pinto, N. Bourriaud, L. Orta, \& M. Damianovic. Lucy Orta. Nueva York: Phaidon

Orta, L. (2003b). Interview with Paul Virilio, 1995 (David Wharry, trad.). En R. Pinto, N. Bourriaud, L. Orta, \& M. Damianovic. Lucy Orta. Nueva York: Phaidon

Pinto, R. (2003). Collective Intelligence: the Work of Lucy Orta. En R. Pinto, N. Bourriaud, L. Orta, \& M. Damianovic. Lucy Orta. Nueva York: Phaidon

Quinn, B. (2003). Intimate Distances: Space, Society, Humanity and Hope - The Work of Lucy Orta. En C. Smith y L. Orta (eds.). Lucy Orta: Body Architecture. Silke Schreiber Verlag. Nueva York: Bloomsbury.

Quinn, B. (2002). Techno Fashion. Nueva York: Bloomsbury.

Studio Orta. (27 marzo, 2013). Lucy + Jorge Orta: Fabulae Romanae [Archivo de video]. Recuperado de https://www.youtube.com/watch?time_continue $=1589 \& \mathrm{v}=$ SEATB_NBBx4 \&feature=emb_logo

The Mad Housers Inc. (s.f.). The Mad Housers [Blog]. Recuperado 10 de diciembre 2019, de http://madhousers.org

UN-Habitat (2017, 4 de julio). UN-Habitat joins forces with partners to address living housing needs of refugees, migrants. Recuperado 10 de noviembre 2019, de https://unhabitat.org/unhabitat-joins-forces-with-partners-to-address-living-housing-needs-of-refugees-migrants Vyzovity, S. (2003). Folding Architecture: Spatial, Structural and Organizational Diagrams. Amsterdam: BIS Publishers.

Vyzovity, S. (2012). Pleat and Play. En Maria Vogiatzaki y Konstantinos-Viktor Spyridonidis (Eds.). Rethinking the Human in Technology Driven Architecture ENHSA-EAAE No55 - Proceedings of the International Conference on Rethinking the Human in Technology Driven Architecture. ENHSA and the European Association for Architectural Education. Amsterdam: BIS Publishers.

\begin{abstract}
This article aims to evaluate the approach to clothing design by the artistic tandem formed by Lucy and Jorge Orta for the development of prototypes of portable shelters that were born thirty years ago in order to cover refugee needs in situations of destitution and migration. The operational and functional basis of a work that was developed in parallel to an environment of reflection and action will be reviewed that, thanks to creators such as Tess Giberson, Vexed Generation or Hussein Chalayan, passed through the sphere of art, fashion and architecture through different performance parameters.
\end{abstract}

Keywords: fashion design - hybridization - architectural design - transdisciplinary - refuge - homelessness - migration - prototypes - portable. 
Resumo: Este artigo tem como objetivo avaliar a abordagem ao design de roupas pelo tandem artístico formado por Lucy e Jorge Orta para o desenvolvimento de protótipos de abrigos portáteis que nasceram há trinta anos para atender às necessidades de refugiados em situação de miséria e migração. Será revista a base operacional e funcional de um trabalho que se desenvolveu paralelamente a um ambiente de reflexão e ação que, graças a criadores como Tess Giberson, Vexed Generation ou Hussein Chalayan, passou pela esfera da arte, moda e arquitetura por através de diferentes parâmetros de desempenho.

Palavras chave: design de moda - hibridização - projeto arquitetônico - transdisciplinar refúgio - sem-teto - migração - protótipos - portátil.

[Las traducciones de los abstracts fueron supervisadas por el autor de cada artículo] 